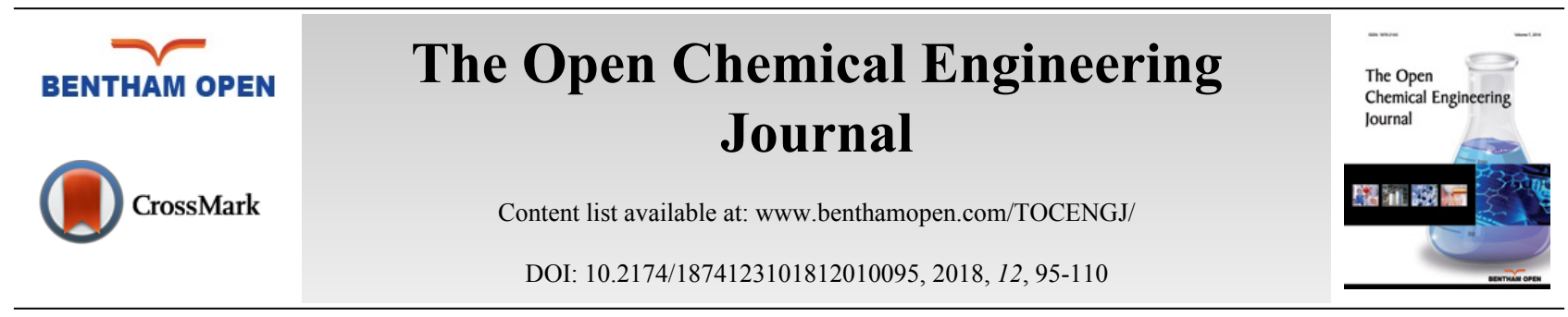

RESEARCH ARTICLE

\title{
Biodiesel Synthesis Monitoring using Near Infrared Spectroscopy
}

\author{
Estela Kamile Gelinski, Fabiane Hamerski, Marcos Lúcio Corazza and Alexandre Ferreira Santos ${ }^{*}$ \\ Department of Chemical Engineering, Federal University of Paraná (UFPR), PO Box 19011, CEP 81531-990, \\ Curitiba, PR, Brazil
}

Received: July 24, 2018

Revised: August 23, 2018

Accepted: September 31, 2018

\begin{abstract}
:
Objective:

Biodiesel is a renewable fuel considered as the main substitute for fossil fuels. Its industrial production is mainly made by the transesterification reaction. In most processes, information on the production of biodiesel is essentially done by off-line measurements.
\end{abstract}

\begin{abstract}
Methods:
However, for the purpose of control, where online monitoring of biodiesel conversion is required, this is not a satisfactory approach. An alternative technique to the online quantification of conversion is the near infrared (NIR) spectroscopy, which is fast and accurate. In this work, models for biodiesel reactions monitoring using NIR spectroscopy were developed based on the ester content during alkali-catalyzed transesterification reaction between soybean oil and ethanol. Gas chromatography with flame ionization detection was employed as the reference method for quantification. FT-NIR spectra were acquired with a transflectance probe. The models were developed using Partial Least Squares (PLS) regression with synthetic samples at room temperature simulating reaction composition for different ethanol to oil molar ratios and conversions. Model predictions were then validated online for reactions performed with ethanol to oil molar ratios of 6 and 9 at $55^{\circ} \mathrm{C}$. Standard errors of prediction of external data were equal to $3.12 \%$, hence close to the experimental error of the reference technique $(2.78 \%)$, showing that even without using data from a monitored reaction to perform calibration, proper on-line predictions were provided during transesterification runs.
\end{abstract}

\section{Results:}

Additionally, it is shown that PLS models and NIR spectra of few samples can be combined to accurately predict the glycerol contents of the medium, making the NIR spectroscopy a powerful tool for biodiesel production monitoring.

Keywords: Biodiesel, Transesterification reaction, NIR spectroscopy, On-line monitoring, Petroluim diesel, Electromagnetic radiation spectrum, HPLC.

\section{INTRODUCTION}

Biodiesel is a renewable fuel [1 - 5] considered the main substitute of fossil fuels [1, 6, 7]. It presents reduced exhaust emissions in comparison to petroleum diesel [8 - 10], it is biodegradable $[2,4,9,11,12]$, and it is miscible in all proportions to petroleum diesel [9]. Similar properties of diesel make possible the use of blends between biodiesel and diesel in combustion engines without any modification $[1,7]$.

Industrial biodiesel production is mainly performed by the transesterification reaction of triacylglycerol (TAG) found in vegetable oils and animal fats, with a short chain alcohol like methanol and ethanol in the presence of a catalyst, producing glycerol as a by-product [1, 5, 6, 12 - 17]. Transesterification reaction occurs in steps producing intermediates like monoacylglycerol (MAG) and diacyglycerol (DAG), which can remain at the end of reaction along

\footnotetext{
*Address correspondence to this author at the Department of Chemical Engineering, Federal University of Paraná (UFPR), PO Box 19011, CEP 81531-990, Curitiba, PR, Brazil; Tel: +55 413361 3590; E-mail: alexfsantos@ufpr.br
} 
with non-reacted TAG and alcohol $[3,14,16,18]$, being contaminants to the produced biodiesel.

In most processes, information on biodiesel production is found essentially through off-line measurements of ester contents of samples taken from the reactor. However, for control purposes, where online process monitoring of biodiesel conversion is required, this is not a satisfactory approach. The most used methods for biodiesel analysis and monitoring are Gas Chromatography (GC) and High Performance Liquid Chromatography (HPLC) [4, 8, 10, 17, 19]. These methods are quite accurate and provide excellent quantitative results, but they are time consuming techniques and involve high costs $[1,3,10,16,18$ - 21]. Infrared spectroscopy has been used as an alternative technique to overcome the disadvantages of off-line methods used in quantification. This technique can still provide accurate results [10, 20], is non-destructive $[10,14,22,23]$, sample pretreatment is not required [10, 23, 24] and it has the advantage of being capable of collecting spectral data online and in-situ [14, 24] demonstrating its ability as a real time monitoring technique.

Infrared spectroscopy refers to the energy in the region of electromagnetic radiation spectrum, and it can be divided into three areas: Near-infrared (NIR), Mid-infrared (MIR) and Far-infrared (FIR) [10]. NIR spectroscopy is a wellestablished analytical technique based on the absorption of electromagnetic energy in the region from 780 to $2500 \mathrm{~nm}$ $\left(12820-4000 \mathrm{~cm}^{-1}\right)[3,13,18]$ that generates spectra with overlapping peaks and valleys according to the complex structure of compounds found in the samples.

Chemometric methods are able to process enormous amounts of sophisticated experimental data that are provided by NIR technique [25], and these methods use statistical or mathematical treatments to extract information about a chemical system from the large data generated by NIR spectroscopy measurements [24]. Partial Least Squares (PLS) has been the main multivariate method used in chemometrics [22, 24 - 26] to develop a calibration model between reference experimental values and spectral data.

When applied specifically to biodiesel analysis, methods based on MIR or NIR spectroscopy and PLS have been developed for biodiesel/diesel blends adulteration monitoring [6, 7, 32, 11, 15, 23, 27 - 31], biodiesel quality or contaminants determination [13, 16, 33 - 35], physical properties estimation, as density and viscosity [1, 36, 37], transesterification reaction monitoring [4, 5, 43, 44, 14, 18, 21, 38 - 42] and quality determination of biodiesel/blends by portable infrared equipment measurements $[20,45,46]$, demonstrating even its use for industrial application. Works based on transesterification monitoring are mainly focused on methyl esters $[3,4,15,18,38,42$, 43], however, even methanol actually being short chain alcohol is used due to its low cost and high reactivity [44 - 48]. There has been a growing interest in the biodiesel produced from ethanol. It is obtained from a renewable source and presents some advantages over the methylic biodiesel, such as higher miscibility with the oil, improved cold flow properties, lower greenhouse gases emission and present higher lubricity when compared to methylic biodiesel [49]. In general, works using NIR spectroscopy are based on calibration models built and applied to samples under well-controlled conditions. The standard experimental procedure generally involves sample collection during reaction, sample neutralization and off-line characterization, for a number of transesterification runs carried out at different molar ratios and catalyst concentrations $[4,5,12,14,18,21]$. These studies involve cumbersome and time-consuming experimental procedures. Efforts to develop robust models based on a small set of samples should still be made to facilitate the transition from laboratory (off-line) data to in-situ applications, as addressed in this paper.

The main objective of this work was the development of a calibration model based on gas chromatography reference measurements combined with NIR and chemometrics for monitoring the biodiesel production from the alkali-catalyzed transesterification of soybean oil with ethanol. Additionally, the quantification of glycerol content using NIR was also addressed. Ester content evaluation and consequently the conversion and quality of the product were the main control variable considered in this study.

\section{EXPERIMENTAL}

\subsection{Biodiesel Production by Transesterification}

Ethyl ester was prepared to perform an alkali-catalyzed transesterification of soybean oil and ethanol in accordance with the procedures described elsewhere [50]. The chemicals used were a commercial soybean oil (Leve, acidity 0.20 $\mathrm{mg} \mathrm{KOH} / \mathrm{g}$, density at $20{ }^{\circ} \mathrm{C} 0.919 \mathrm{~g} / \mathrm{cm}^{3}$ ), ethanol (Neon, $99.8 \%$ ) and sodium hydroxide (Exodo, >97\%). All chemicals were used as received. Reactions were carried in a $250 \mathrm{~mL}$ three-neck glass equipped with a thermocouple, reflux column and stirred with a magnetic bar. The system was kept in a glycerol bath over a heater with a magnetic stirrer. 
An ethanol to oil molar ratio of $6: 1$ was used with $0.6 \mathrm{wt} \%$ of catalyst related to the oil mass $[3,14,38,51]$. Six transesterification runs were conducted in a batch where the mixtures of ethanol and sodium hydroxide were added to the reactor and kept under stirring until complete solubilization of the sodium hydroxide, and then the oil was added, keeping the temperature at $55^{\circ} \mathrm{C}$ during $45 \mathrm{~min}$. The total volume of the reactor was transferred to a separation funnel where the lower phase was removed. The upper phase was washed with a $5 \mathrm{wt} \%$ solution of $\mathrm{HCl} \mathrm{P.A.} \mathrm{(Vetec)} \mathrm{at} 60{ }^{\circ} \mathrm{C}$, discarding the lower phase and washing again two times with distilled water at $60{ }^{\circ} \mathrm{C}$.

The ester rich-phase obtained after washing steps was rotary evaporated at $60{ }^{\circ} \mathrm{C}$ during 40 min to remove the remaining ethanol. Ten percent of the mass was considered as non-reacted oil, adding ethanol and $\mathrm{NaOH}$ keeping the original molar ratio and the catalyst amount, proceeding the second step of the transesterification at the same conditions of the first step. The rotary evaporated product was kept on a stove at $60{ }^{\circ} \mathrm{C}$ during $16 \mathrm{~h}$ and then it was filtered through sodium sulfate P.A. min. 99\% (Vetec) and qualitative filter paper. Produced biodiesel was analyzed by gas chromatography and stored on an amber flask under refrigeration [21].

\subsection{Synthetic Samples Preparation}

A total of 29 synthetic samples containing soybean oil, ethanol, ethyl ester (produced and analyzed as described above) and glycerol in different proportions were prepared based on different ethanol to oil molar ratios and conversions, simulating the composition in the reaction medium for a soybean oil and ethanol transesterification (without taking into account the catalyst presence in the reaction medium). The molar ratio of $6: 1$ was chosen to simulate conversion increments of $10 \%$, generating 11 samples varying from 0 to $100 \%$ conversion. Molar ratios of 3:1, 9:1 and 12:1 had conversion increments of $20 \%$, generating each one 6 samples from 0 to $100 \%$ conversion. The samples were analyzed by gas chromatography and stored in closed flasks and kept under refrigeration.

\subsection{Quantitative Analysis}

The glycerol content was determined following the method described by Bondiolli and Bella (2005) [52] and contents of ethyl esters, free fatty acids and TAG were determined by gas chromatography (GC) with flame ionization detector based on the European standard EN 14105.

Compound identification for GC method was done based on the retention time of standards belonging to several classes of compounds of interest. Quantification was performed after the instrument calibration with reference substances and internal standards. All the reference standards used to build the calibration curves (glycerol, oleic acid, ethyl linoleate, monoolein, diolein and triolein) were $>99 \%$ purity (Sigma-Aldrich).

Prior to chromatographic analysis all samples were silylated with N-metil-N-trimetilsiltrifluoracetamida (MSTFA)(Sigma-Aldrich, 98.5\%)and diluted with heptane (Sigma-Aldrich, 99\%). The concentration of the injection solution was approximately $1 \mathrm{mg} / \mathrm{mL}$.

All samples were analyzed using a Shimadzu chromatograph (GC 2010 Plus), a capillary column Select biodiesel (15 m x $0.32 \mathrm{~mm} \times 0.10 \mu \mathrm{m}$, Agilent), flame ionization detector (FID) and split injection mode (1:10) for $1 \mu \mathrm{L}$ of the solution. The injection temperature of $380{ }^{\circ} \mathrm{C}$ was used for quantification. The injector and detector temperatures were $380{ }^{\circ} \mathrm{C}$ and $400{ }^{\circ} \mathrm{C}$, respectively. The oven temperature was programmed to increase from $50{ }^{\circ} \mathrm{C}$ to $180{ }^{\circ} \mathrm{C}$ with a 15 ${ }^{\circ} \mathrm{C} / \mathrm{min}$ rate. The heating rate was reduced to $7{ }^{\circ} \mathrm{C} / \mathrm{min}$ and the temperature was raised to $230{ }^{\circ} \mathrm{C}$, the final temperature of $380{ }^{\circ} \mathrm{C}$ was achieved with a $10{ }^{\circ} \mathrm{C} / \mathrm{min}$ rate, keeping this temperature during $6 \mathrm{~min}$. The total time of analysis was $36.81 \mathrm{~min}$ and helium analytical grade (White Martins) was used as mobile phase. The maximum experimental error of this technique with respect to the ester content determination was $2.78 \%$.

\subsection{NIR Spectra Acquisition}

The acquisition of the near-infrared spectra was performed in a Vertex 70 (Bruker) spectrophotometer. The spectra were acquired using a Fourier transform transflectance immersion probe, in the wavenumber range from 4000 to 10000 $\mathrm{cm}^{-1}$, with a resolution of $4 \mathrm{~cm}^{-1}$ and 32 scans. The optical path of the accessory was equal to 2.5 mm, measurements were made at $20{ }^{\circ} \mathrm{C}$ for synthetic samples and at reaction temperature when the transesterification was monitored, both of them with stirring because the formation of a two-phase system is very fast. The background spectrum was acquired with the empty probe and the NIR data were obtained using OPUS 4.0 software provided with the FT-NIR instrument. 


\subsection{On-line Reaction Monitoring}

For on-line reaction monitoring, a mixture of ethyl esters was produced according to the procedure described in section 2.1, following only the first step of the reaction. The first reaction (R6) was performed with ethanol to oil molar ratio of 6:1 and at each $2 \mathrm{~min}$, starting from $6 \mathrm{~min}$ of reaction time, NIR spectrum was acquired at the same time an aliquot of the reaction medium was collected, generating a total of 9 aliquots. The second reaction (R9) was performed with ethanol to oil molar ratio of 9:1, collecting NIR spectrum and an aliquot of reaction medium at each 2 min starting from 2 min of reaction time, generating a total of 14 aliquots. All the aliquots were instantly neutralized with glacial acetic acid and analyzed by GC.

\subsection{Data Analysis and Calibration Model}

MATLAB R2016a software was used to perform the data analysis (spectra pre-processing) to calibrate and validate the regression model. Outlier detection was performed based on leverage and studentized y-residuals values. Basically, an outlier was identified if its leverage value was 2.5 times above the average leverage value or if its studentized yresidual value was higher than 2.5 .

All spectra were mean centered before the modeling procedures. The region with the greatest spectral differentiation was identified and used to develop a multivariate calibration model based on partial least squares regression. First and second order Savitzky-Golay derivatives with different segment sizes were tested as spectra pre-processing techniques. Intending to monitor the transesterification reaction, six different models $(\mathrm{M})$ based on the ethyl ester content prediction were developed using synthetic samples data according to ethanol to oil molar ratios. M1: calibrated with synthetic samples of molar ratios 3, 6, 9 and 12; M2: calibrated with synthetic samples of molar ratios 3,6 and 9; M3: calibrated with synthetic samples of molar ratios 6, 9 and 12; M4: calibrated with synthetic samples of molar ratios 6 and 9; M5: calibrated with synthetic samples of molar ratio 6; M6: calibrated with synthetic samples of molar ratio 9. These models were selected because they involved the molar ratios used for on-line monitored reactions.

The best number of Latent Variables (LV) was detected by the root mean square error of cross-validation (RMSECV), using full cross-validation method, enabling that all the samples were tested at least once as external data. Model validation was made by the Root Mean Square Error of Prediction (RMSEP) using data from two base catalyzed transesterification reactions monitored on-line that were not used during the calibration step.

\section{RESULTS AND DISCUSSION}

\subsection{Quantification}

During sample preparation previously to the $\mathrm{GC}$ analysis, ethanol was removed in an air circulating oven at $60{ }^{\circ} \mathrm{C}$ and then gravimetrically quantified. Glycerol was not detected during GC analysis probably due to decantation during sample preparation, for this reason, glycerol quantification was performed by UV-Vis spectroscopy.

Since we used synthetic mixtures involving biodiesel, ethanol, soybean oil and glycerol, it was possible to compare the GC results with those expected for ester contents according to the mass fraction of synthetic samples, which is the compound of main interest for the construction of the calibration models for transesterification monitoring. Additionally, the results for triacylglycerol (representing the soybean oil), ethanol and glycerol content are also shown below. Figs. (1 and 2) show the comparison for ester TAG content results, respectively, with the ones expected by the mass fraction of each compound used in the preparation of the synthetic samples, both analyses were performed by CGFID

From the results presented in Figs. (1 and 2), it is observed that some data show deviations from the expected value. Therefore, during the calibration step of the PLS regression model tests were performed to identify the outliers. Fig. (3) presents the results for the quantification of ethanol by mass evaporation in a heated oven, showing that the results are within the expected range.

Fig. (4) shows the results of glycerol quantification by UV-Vis and it is noted that the majority of the results were below the expected level. The discrepancy regarding the results for the glycerol quantification can be explained by the high density and viscosity of this compound and also due to the glycerol separation from the ester phase, which rapidly decants and remains trapped in the walls of vials and pipette tips. However, this is not unusual and should not be overemphasized. On the other hand, this issue encourages the development of alternative analytical tools able to accurately detect the glycerol contents in the mixture. 


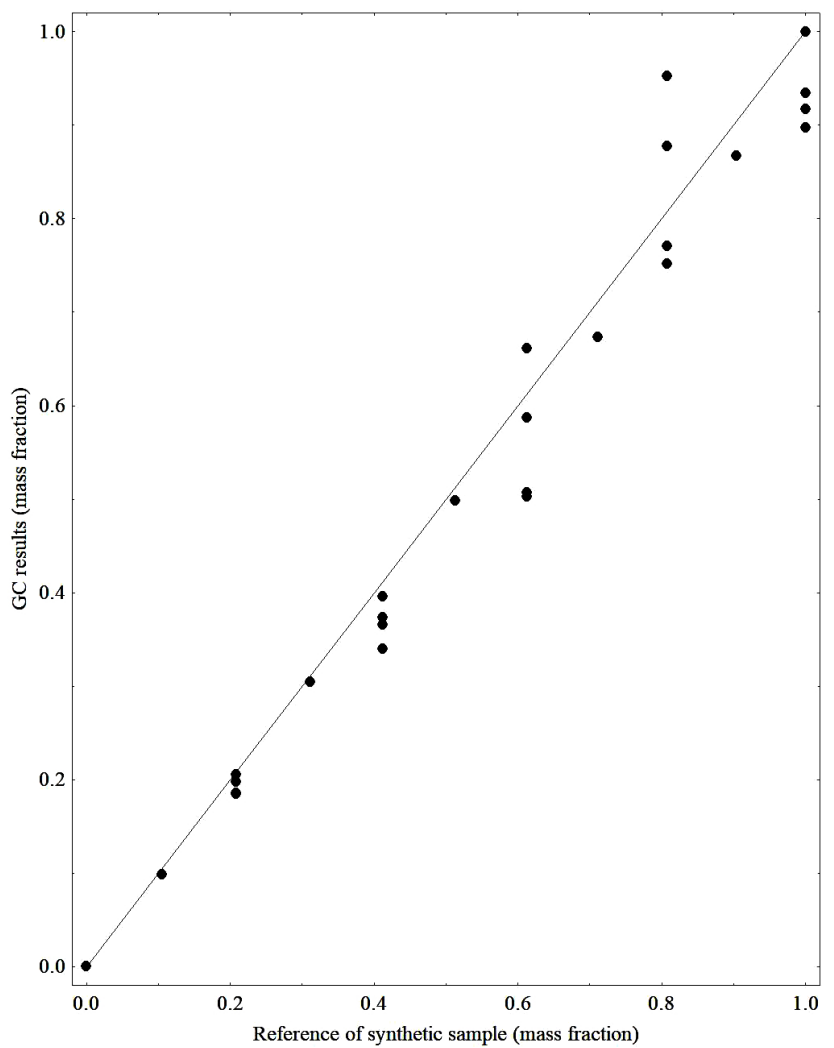

Fig. (1). Ethyl ester content by GC versus ethyl ester content in the synthetic samples.

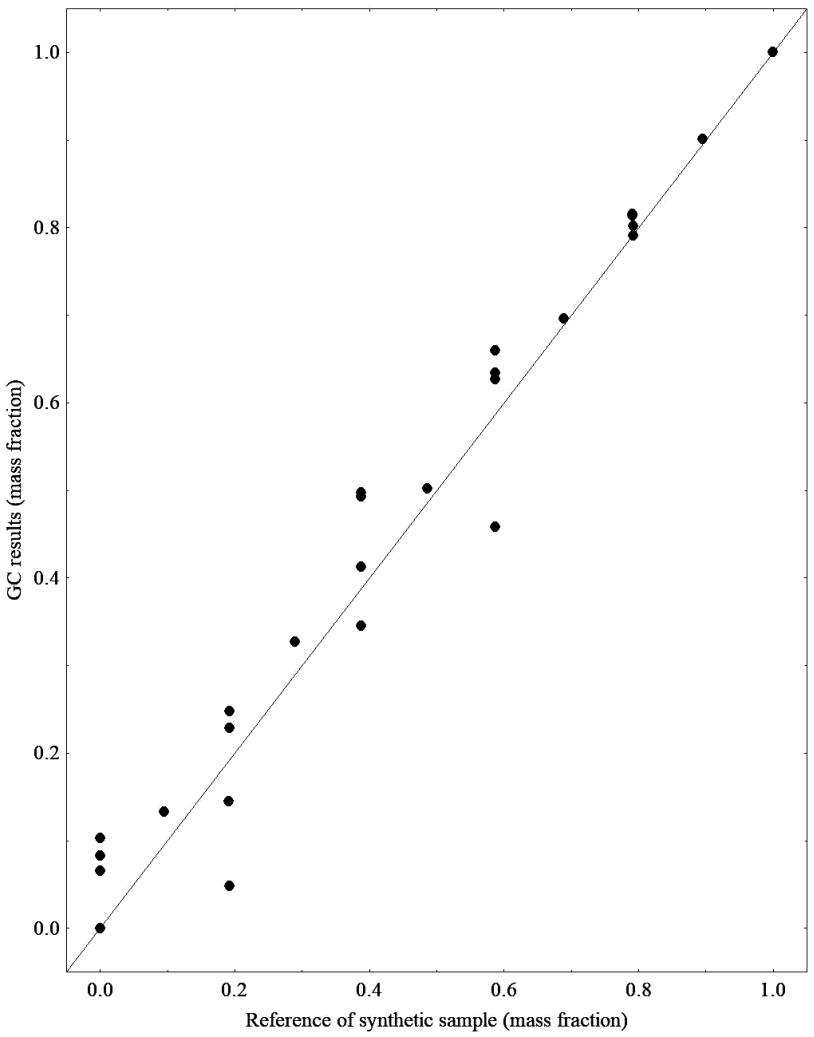

Fig. (2). Triacylglycerol content by GC versus triacylglycerol content in the synthetic samples. 


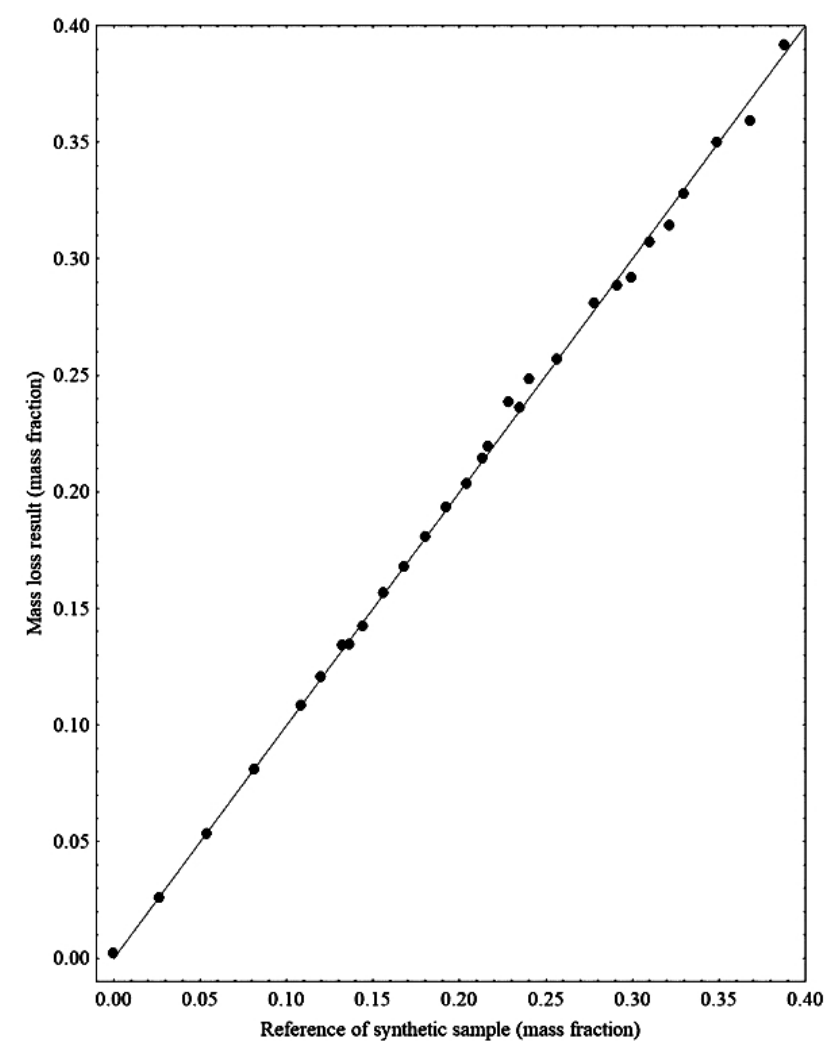

Fig. (3). Ethanol content by mass loss versus ethanol content in the synthetic samples.

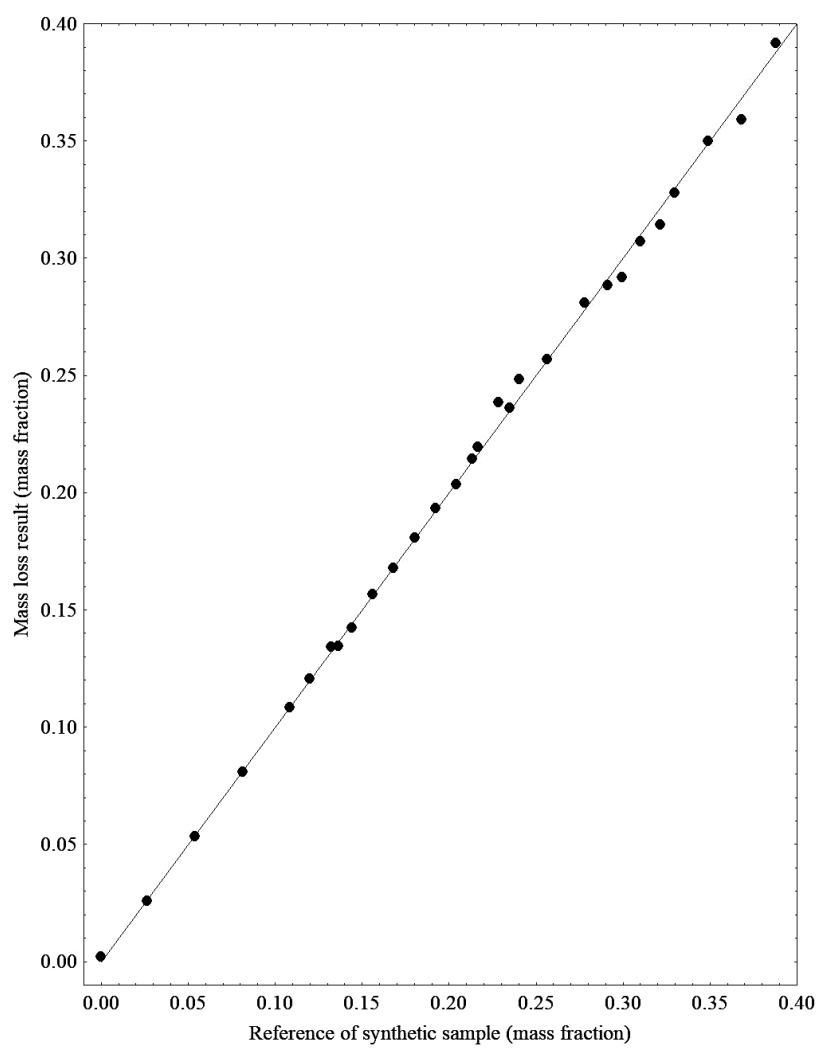

Fig. (4). Glycerol content by UV-Vis method versus glycerol content in the synthetic samples. 


\subsection{NIR Spectra and Multivariate Calibration}

Fig. (5) shows the NIR spectra of the synthetic samples that were used to build the ethyl ester content calibration model. The two extreme wavenumber regions ranging from $10000-9000 \mathrm{~cm}^{-1}$ and $4450-4000 \mathrm{~cm}^{-1}$ presented noisy signal and were not used for the PLS regression model.

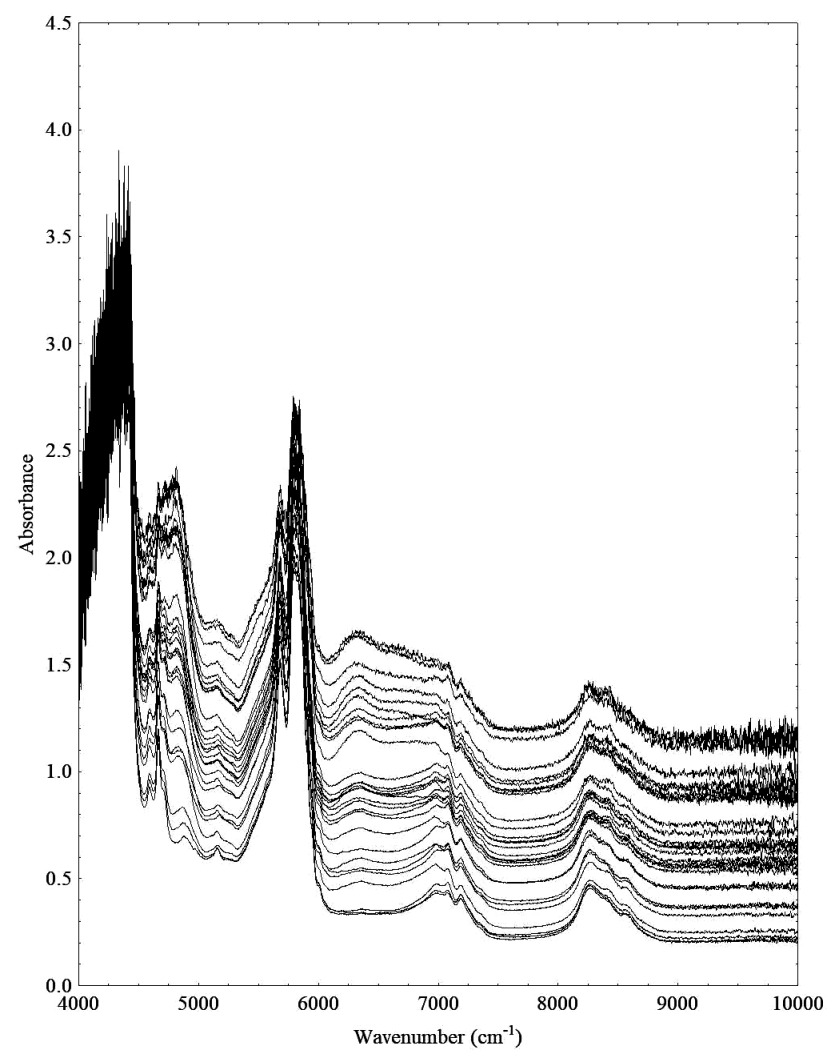

Fig. (5). Synthetic samples NIR spectra without pre-treatment.

\subsubsection{Ester Content Prediction Models}

The first model M1 was developed using the wavenumber region from $9000-4450 \mathrm{~cm}^{-1}$ and according to variable contributions the initial region was reduced, removing wavenumbers with low contributions until lack of model improvement. From all the synthetic samples four data points were detected as outliers following the procedure in section 2.6 and removed from calibration models.

The wavenumber region from $6150-5941 \mathrm{~cm}^{-1}$ showed the highest spectral differentiation to build the calibration models. In fact, this region corresponds to the first overtone of $\mathrm{C}-\mathrm{H}$ stretching for $\mathrm{CH}_{2}$ and $\mathrm{CH}_{3}$ bonds, and to the best of our knowledge the wavenumber region used in this work was not tested yet for transesterification reaction monitoring and is narrower than the regions tested by Lima et al. [14] and Richard et al. [5, 21], which is advantageous because narrow bands can be detected by smaller and portable infrared instruments.

First and second order Savitzky-Golay derivatives with different segment sizes and polynomial order of 2 and 3 were tested to check the model improvement for R6 prediction, however, the results were worse than the ones using raw spectra and in some cases, this pre-treatment generated models with RMSEP values above 10\%, as presented in Table $\mathbf{1}$. The use of only mean-centered spectra is not usual because most of the works using NIR aiming models development for transesterification monitoring have used at least a derivative pre-processing technique [4, 5, 14, 18, 21].

Table 1. Results for M1 model without and with SG derivative spectral pre-treatment for 25 calibration points and 9 external validation points.

\begin{tabular}{|c|c|c|c|c|c|c|c|}
\hline Derivative order & - & \multicolumn{4}{|c|}{1} & \multirow{2}{*}{\multicolumn{2}{|c|}{$\frac{2}{2 \text { or } 3}$}} \\
\hline Polynomial order & - & & & & & & \\
\hline Segment size & - & 11 & 21 & 11 & 21 & 11 & 21 \\
\hline
\end{tabular}


(Table $\square$ ) contd.....

\begin{tabular}{|c|c|c|c|c|c|c|c|}
\hline Derivative order & - & \multicolumn{4}{|c|}{1} & \multirow{2}{*}{\multicolumn{2}{|c|}{$\frac{2}{2 \text { or } 3}$}} \\
\hline Polynomial order & - & \multicolumn{2}{|c|}{2} & \multicolumn{2}{|c|}{3} & & \\
\hline $\mathbf{L V}$ & 4 & 6 & 3 & 6 & 3 & 6 & 4 \\
\hline RMSEC (\%) & 2.83 & 1.97 & 3.58 & 2.42 & 3.46 & 3.02 & 3.45 \\
\hline RMSECV (\%) & 5.39 & 4.88 & 4.57 & 5.14 & 5.75 & 7.91 & 6.41 \\
\hline RMSEP (\%) & 4.59 & 5.80 & 6.76 & 9.99 & 7.95 & 12.89 & 11.03 \\
\hline
\end{tabular}

The same procedure used to create the M1 model was performed for the other models always using the wavelength region of $6150-5941 \mathrm{~cm}^{-1}$ for the calibration and using the data from the on-line monitored reactions for model validation. Predicted values for ester content versus reference values (CG-FID analysis) are shown in Fig. (6).
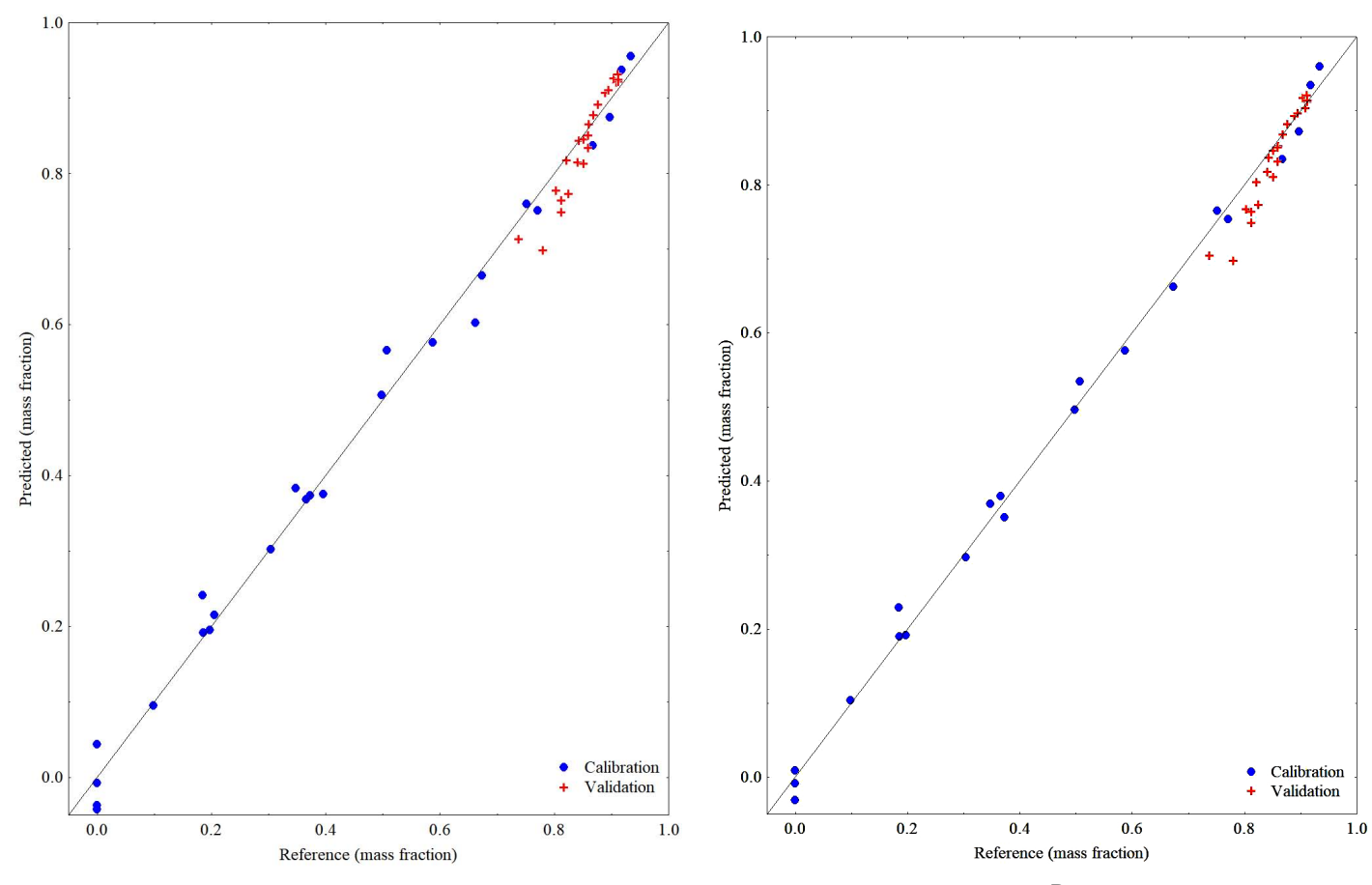

\$ए
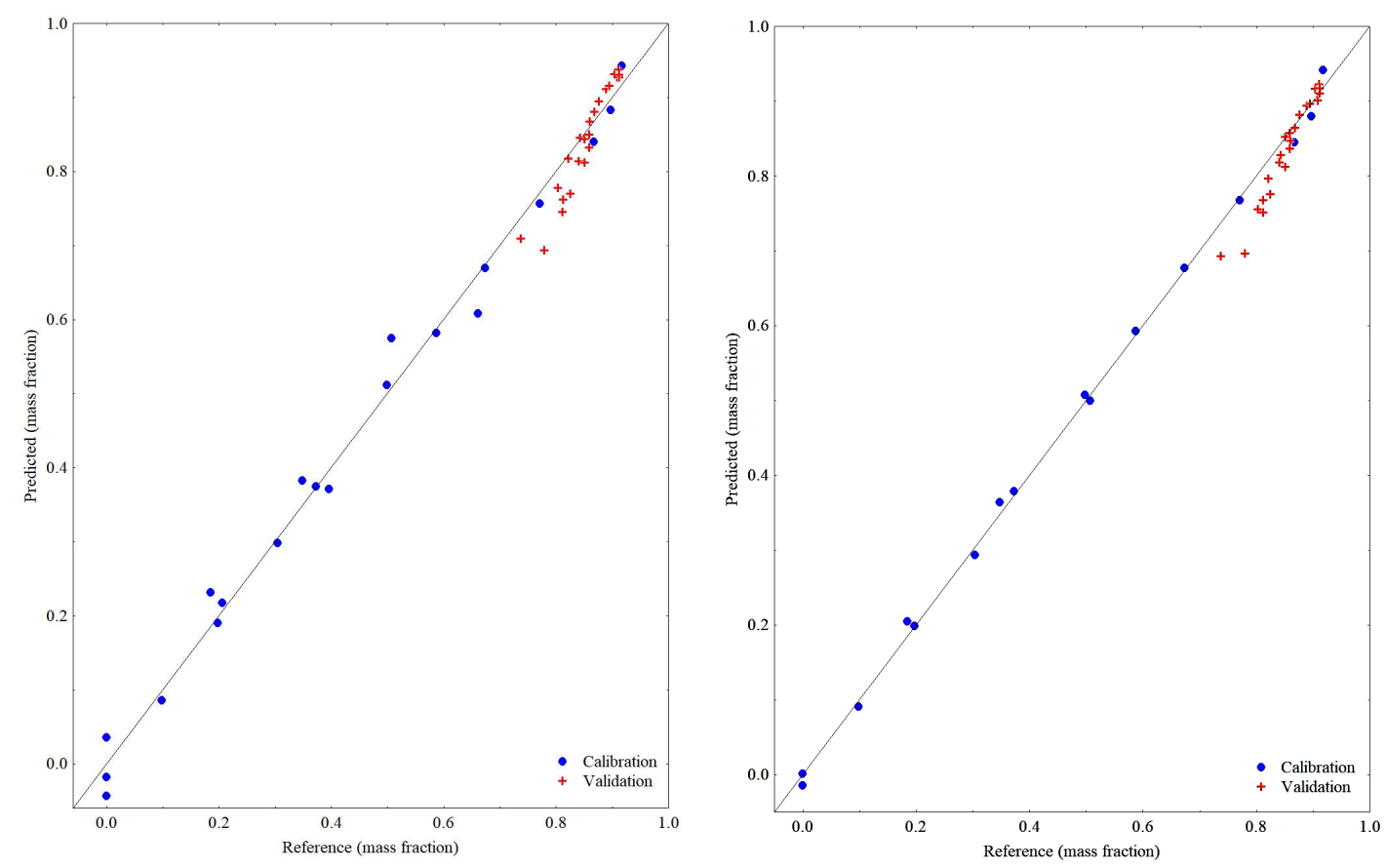

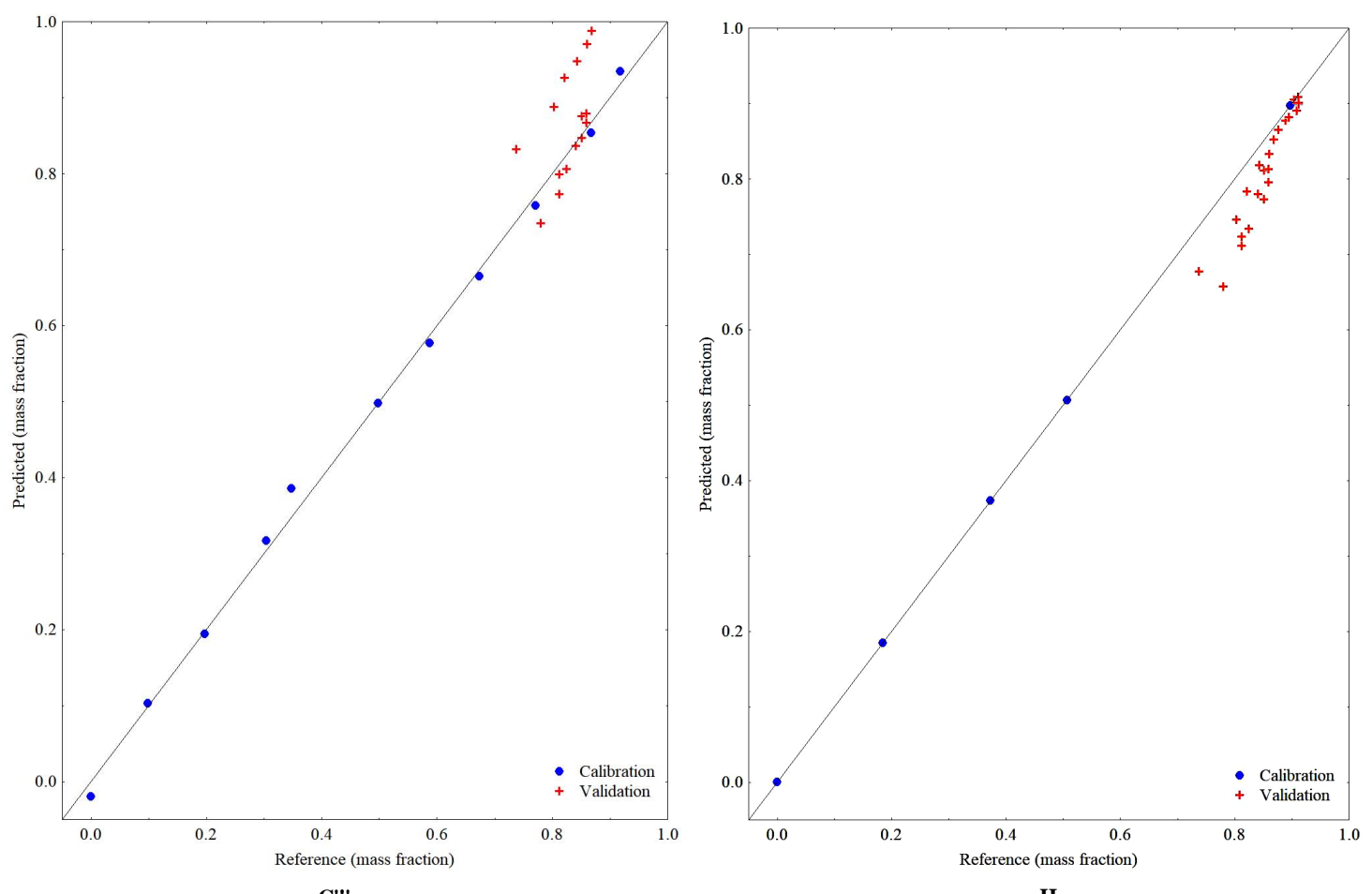

( $\square$

)

Fig. (6). Predicted versus measured plot of PLS models for ethyl ester content, validated with (R6 + R9) data set using different proposed models: a) M1 b) M2 c) M3 d) M4 e) M5 f) M6.

Table 2 summarizes the results of PLS regression models developed with $6150-5941 \mathrm{~cm}^{-1}$ wavenumber region and different number of calibration points $(\mathrm{N})$ for ethyl ester content. All of these models were validated using data from two sets of alkali-catalyzed transesterification reaction (R6 and R9).

Table 2. PLS results for ethyl ester content calibration and prediction.

\begin{tabular}{|l|c|c|c|c|c|}
\hline Model & M1 & M2 & M3 & M4 & M5 \\
\hline N & 25 & 21 & 20 & 16 & 11 \\
\hline LV & 4 & 4 & 4 & 5 & 2 \\
\hline RMSEC (\%) & 2.83 & 2.01 & 2.94 & 1.30 & 1.60 \\
\hline RMSECV (\%) & 5.39 & 4.04 & 6.10 & 4.47 & 2.21 \\
\hline R $^{2}$ & 0.9916 & 0.9959 & 0.9952 & 0.9966 & 0.9931 \\
\hline RMSEP (\%) R6 & 4.59 & 4.63 & 4.82 & 4.44 & 2.41 \\
\hline RMSEP (\%) R9 & 1.55 & 1.53 & 1.90 & 2.04 & 11.59 \\
\hline RMSEP (\%) R6+R9 & 3.12 & 3.13 & 3.36 & 3.13 & 2.86 \\
\hline
\end{tabular}

Based on the RMSEP values generated by the models, M1, M2, M3 and M4 models are quite accurate as the reference method used, and an F-test at $95 \%$ of confidence level stated that the four results are statistically the same, therefore any of them can be used for on-line monitoring of transesterification reaction with satisfactory precision. Taking into account the RMSEP for R6 plus R9, the best prediction for on-line monitoring the transesterification reactions will be guaranteed using model M1. However, model M4 can be used if one requires a better prediction for R6 reaction with relatively good results for reaction $\mathrm{R} 9$, since this model spent less chemicals and time of analysis due to the lower number of samples used to develop the model.

As expected the models M5 and M6 are only accurate for the molar ratio of reactants in which each model was calibrated. Despite the sensitivity of the spectral data to the ester contents, it is hard to a model being able to predict the ester content in the mixture at conditions far from the ones adopted during the calibration step, and normally the models will be valid for the data range of the dependent variable. Unfortunately, the data set of synthetic samples simulating a reaction with molar ratio of 9 has only 5 data, leading to a model that is not the best one for this molar ratio, as opposed 
to the model developed with synthetic samples simulating a reaction with molar ratio of 6 that generated the best model to predict the reaction performed with this molar ratio.

Richard et al. [21] developed a model for ethyl ester content prediction using NIR reflectance measurement and the results were $\mathrm{RMSEC}=4.08 \%$ and $\mathrm{RMSEP}=4.10 \%$, with 5 latent variables and 44 calibration samples for a set of molar ratios of $6,9,16.2,22.7$ and 45.4 , at a reaction temperature of $65{ }^{\circ} \mathrm{C}$. In the current work, the M1 model presented lower RMSEP values for ethyl ester content prediction for reactions performed with molar ratios of 6 and 9 , with only 4 latent variables and less calibration samples. The predicted content of ethyl ester showed no significant deviation from the reference data as shown in Fig. (6A) for the M1 model.

Works for methyl or ethyl ester content prediction during a transesterification reaction, calibrated and validated with only one alcohol to oil molar ratio were developed by Lima et al. [14], obtaining RMSEP $=1.51 \%$ with 6 latent variables and 50 calibration samples, and also in the study presented by Pinzi et al. [18] obtained RMSEP $=2.55 \%$ with 3 latent variables and 79 calibration samples, and Richard et al. [21] reached a RMSEP $=3.52 \%$ for 9 latent variables and 40 samples. In the present work, there are two models calibrated with data from only one molar ratio, the models M5 and M6 which generated RMSEP $=2.41 \%$ for molar ratio of 6 and RMSEP $=2.86 \%$ for molar ratio of 9 . These results are quite good, comparable with the results presented by Pinzi et al. [18] for a methyl ester system, nevertheless, we have used a much lower number of calibration samples.

Besides the final ester content estimation in a transesterification reaction, the developed PLS model can be used for motoring the kinetics of the transesterification reaction based on the on-line monitoring data. Fig. (7) depicts a comparison of estimated values based on the M1 model and the ester content measured by CG-FID for kinetic data at a different molar ratio of reactants. The behavior is similar to the one presented by Richard et al. [21] and the equilibrium condition is reached in few minutes of reaction justifying the use of a technique as fast as NIR associated to PLS regression model for on-line monitoring purposes.

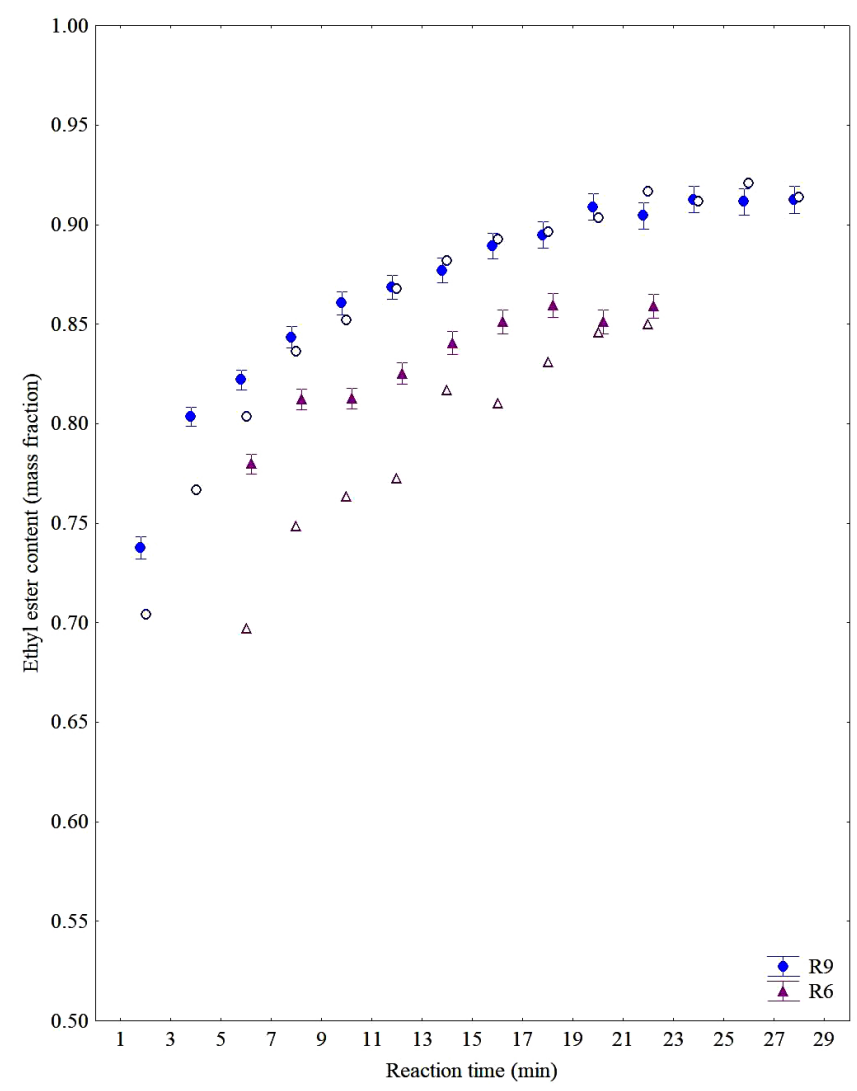

Fig. (7). Ethyl ester content during reaction time for R6 and R9 according to GC reference (R6 - triangles, R9 - circles) and on-line NIR predictions using M1 model (open circle and triangle symbols). 
Using the models that provided the best prediction results for R6 (M5) and the model with best prediction results for R9 (M2), the ethyl ester content during reaction time progress is presented in Fig. (8).

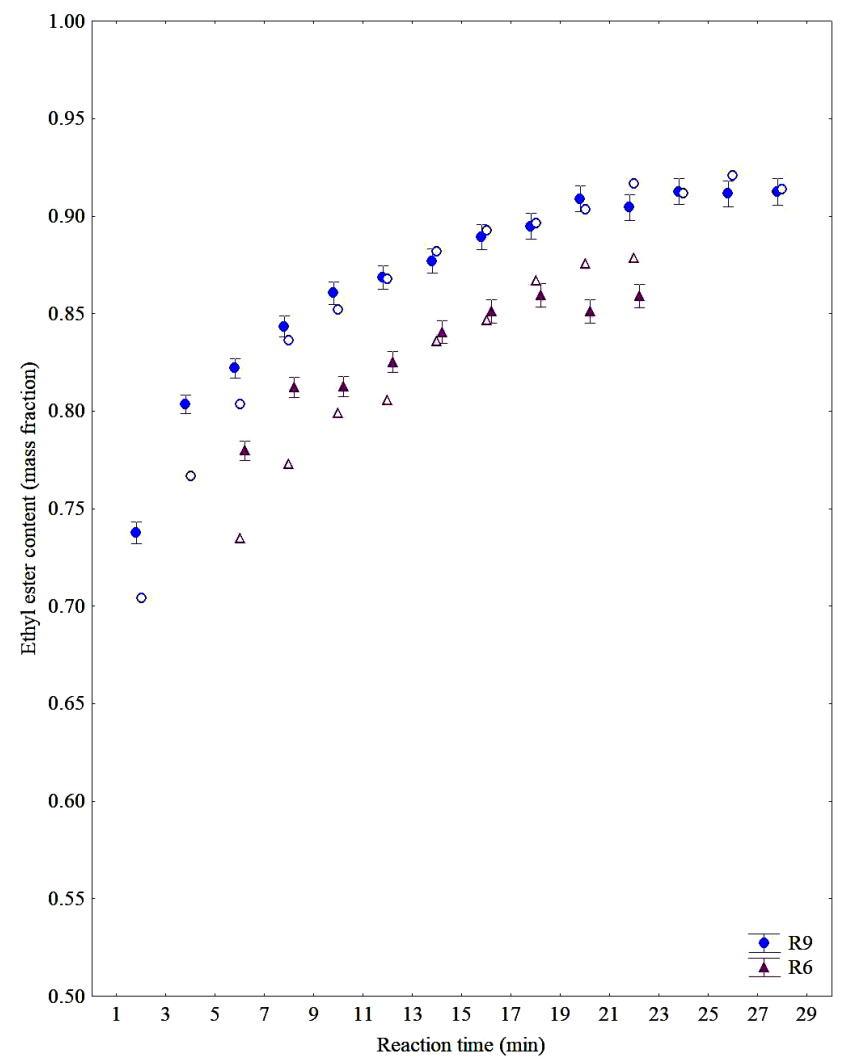

Fig. (8). Ethyl ester content during reaction time for R6 and R9 according to GC reference (R6 - triangles, R9 - circles) and on-line NIR predictions using M5 (open triangles) and M2 (open circles) models.

It can be noted as the best fit for the prediction of ester contents during R6 and R9 reactions using the best models of each reaction (Fig. 8). On the other hand, one can also conclude that a single model (such as M1) that is flexible enough to predict both reactions can also adequately serve the purposes of monitoring (see Fig. 7).

In general, the results regarding the overall ester content analysis achieved in this work are very satisfactory considering the following aspects: first, the models were developed from synthetic samples, while most part of the authors perform the reaction at different conditions, collecting at the same time samples from reaction medium and NIR spectrum, neutralizing samples instantaneously; second, models built for NIR spectra acquired at $20{ }^{\circ} \mathrm{C}$ were able to predict data from a reaction at $55^{\circ} \mathrm{C}$ and third, few samples were necessary, representing laboratory cost saving. Consequently, these results are encouraging, and show that the NIR strategy presented is robust enough and could be applied at actual biodiesel production sites as an on-line monitoring tool applied to optimization of production.

\subsubsection{Glycerol Content Prediction Model}

During the analysis of the synthetic samples, a large deviation between the glycerol results from the UV-Vis analysis and the expected results based on the mass fraction of this compound on the synthetic samples was observed. Since the spectra of the synthetic samples can also be used to develop a calibration model for the glycerol content in the sample, a model was developed, feeding the expected values of glycerol fraction from synthetic samples composition.

In Fig. (9) it can be noticed that most of the results of the glycerol contents of the UV-Vis analysis were below the expected values, as already presented in section 3.1. The prediction model built using PLS regression used the wavenumber range of $5930-6181 \mathrm{~cm}^{-1}, 4$ latent variables, RMSEC of $0.15 \%$, RMSECV of $0.15 \%$ and RMSEP of $0.20 \%$ in relation to the percentage of glycerol in the sample. 


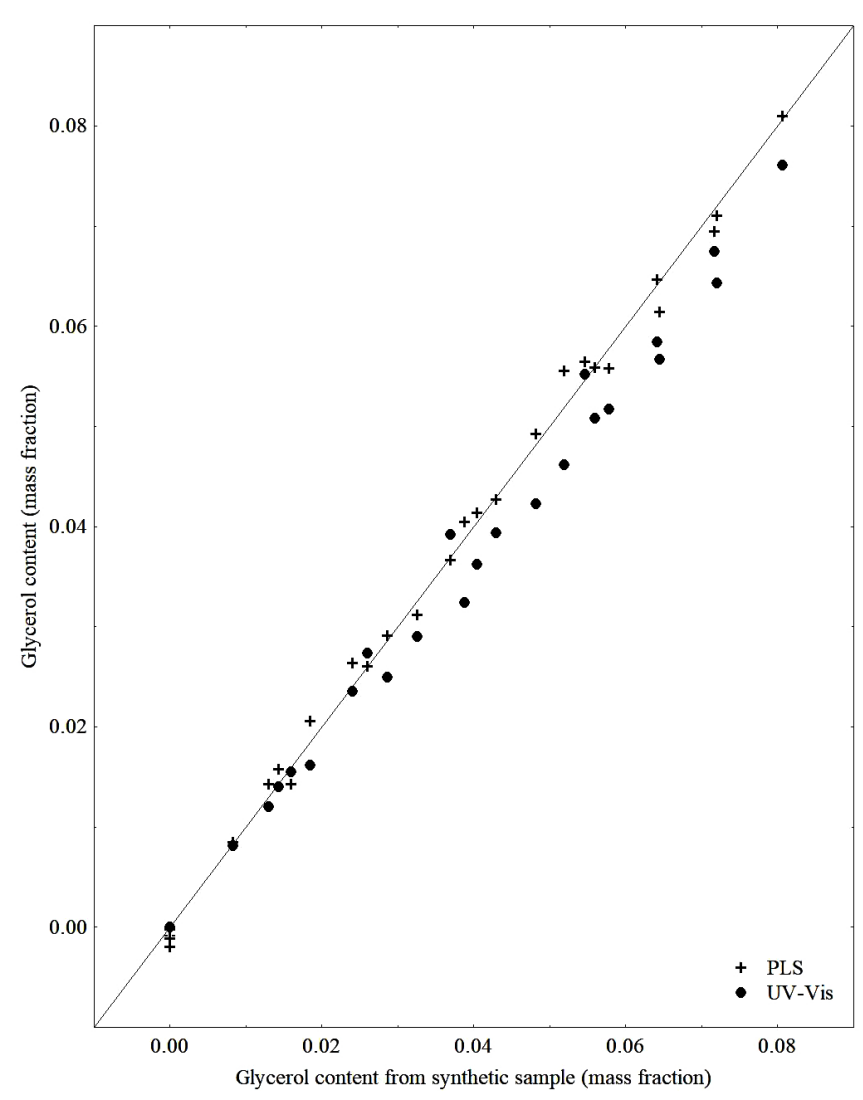

Fig. (9). Glycerol content by PLS calibration model and UV-Vis analysis.

The work of Pinzi et al. (2012) [18], focused on the use of NIR in the monitoring of biodiesel production and that of Dorado et al. (2011) [8], focused on the use of NIR to attest the biodiesel quality, also present models to predict the glycerol content. The Pinzi et al. (2012) model presented RMSECV of $0.56 \%$ in relation to the mass percentage of glycerol present in the sample, with 4 latent variables and RMSEP equal to $0.73 \%$ for validation of the model covering glycerol contents of 0.32 to 3.38 in percentage by mass. Dorado et al. (2011) used a range of glycerol content for calibration ranging from 0.005 to 0.050 in mass percentage and the model associating UV-Vis and NIR presented RMSECV of $0.083 \%$.

\section{CONCLUSION}

Several PLS regression models built with synthetic samples for ethyl ester content prediction, based on NIR measurements, were successfully applied for on-line monitoring of alkali-catalyzed transesterification reactions of soybean oil and ethanol. The calibration strategy involved the preparation of 29 synthetic samples to mimic the biodiesel production at different ethanol to oil ratios, the collection of NIR spectra at room temperature $\left(20^{\circ} \mathrm{C}\right)$ and PLS regression to correlate NIR spectra with the ester content data. The wavenumber range used was narrow $(6150-5941$ $\mathrm{cm}^{-1}$ ) and the calibration model was able to provide proper predictions of ester contents during transesterification reactions performed at $55{ }^{\circ} \mathrm{C}$, with mean error values (3.12\%) close to the deviations attributed to the reference technique $(2.78 \%)$. Additionally, a calibration model was successfully built correlating the glycerol content $(0-8.0$ $\mathrm{wt} \%$ ) in the biodiesel and NIR data, with a standard error of prediction of $0.20 \%$.

Results demonstrated that with relatively few experimental data, calibration models can be developed using off-line measurements, showing that NIR is a fast and accurate technique for quantification, allowing an effective monitoring of the reaction and reducing time and process cost in the ethyl biodiesel production.

\section{CONSENT FOR PUBLICATION}

Not applicable. 


\section{CONFLICT OF INTEREST}

The authors declares no conflict of interest, financial or otherwise.

\section{ACKNOWLEDGEMENTS}

The authors thank the Brazilian funding agencies (CNPq - grants 406737/2013-4, CAPES and Fundação Araucária) for supporting this work and providing scholarships. The authors also thank the Laboratory of Infrared Absorption Spectroscopy of UFPR for the spectrophotometer availability.

\section{REFERENCES}

[1] R.M. Balabin, E.I. Lomakina, and R.Z. Safieva, "Neural network (ANN) approach to biodiesel analysis: Analysis of biodiesel density, kinematic viscosity, methanol and water contents using near infrared (NIR) spectroscopy", Fuel, vol. 90, pp. 2007-2015, 2011. [http://dx.doi.org/10.1016/j.fuel.2010.11.038]

[2] D.T. Chadwick, K.P. Mcdonnell, L.P. Brennan, C.C. Fagan, and C.D. Everard, "Evaluation of infrared techniques for the assessment of biomass and biofuel quality parameters and conversion technology processes : A review", Renew. Sustain. Energy Rev., vol. 30, pp. 672-681, 2014

[http://dx.doi.org/10.1016/j.rser.2013.11.006]

[3] P. Baptista, P. Felizardo, J.C. Menezes, and M.J.N. Correia, "Multivariate near infrared spectroscopy models for predicting the methyl esters content in biodiesel", Anal. Chim. Acta, vol. 607, no. 2, pp. 153-159, 2008. [http://dx.doi.org/10.1016/j.aca.2007.11.044] [PMID: 18190803]

[4] M.H.M. Killner, J.J.R. Rohwedder, and C. Pasquini, "A PLS regression model using NIR spectroscopy for on-line monitoring of the biodiesel production reaction", Fuel, vol. 90, pp. 3268-3273, 2011. [http://dx.doi.org/10.1016/j.fuel.2011.06.025]

[5] R. Richard, Y. Li, B. Dubreuil, S. Thiebaud-Roux, and L. Prat, "On-line monitoring of the transesterification reaction between triglycerides and ethanol using near infrared spectroscopy combined with gas chromatography", Bioresour. Technol., vol. 102, no. 12, pp. 6702-6709, 2011 .

[http://dx.doi.org/10.1016/j.biortech.2011.03.111] [PMID: 21514822]

[6] J.C.L. Alves, and R.J. Poppi, "Biodiesel content determination in diesel fuel blends using near infrared (NIR) spectroscopy and support vector machines (SVM)", Talanta, vol. 104, pp. 155-161, 2013.

[http://dx.doi.org/10.1016/j.talanta.2012.11.033] [PMID: 23597903]

[7] J.C.L. Alves, and R.J. Poppi, "Quantification of conventional and advanced biofuels contents in diesel fuel blends using near-infrared spectroscopy and multivariate calibration", Fuel, vol. 165, pp. 379-388, 2016. [http://dx.doi.org/10.1016/j.fuel.2015.10.079]

[8] M.P. Dorado, S. Pinzi, A. de Haro, R. Font, and J. Garcia-Olmo, "Visible and NIR spectroscopy to assess biodiesel quality: Determination of alcohol and glycerol traces", Fuel, vol. 90, pp. 2321-2325, 2011. [http://dx.doi.org/10.1016/j.fuel.2011.02.015]

[9] T. Issariyakul, and A.K. Dalai, "Biodiesel from vegetable oils", Renew. Sustain. Energy Rev., vol. 31, pp. 446-471, 2014. [http://dx.doi.org/10.1016/j.rser.2013.11.001]

[10] W-B. Zhang, "Review on analysis of biodiesel with infrared spectroscopy", Renew. Sustain. Energy Rev., vol. 16, pp. 6048-6058, 2012. [http://dx.doi.org/10.1016/j.rser.2012.07.003]

[11] D.D.S. Fernandes, A.A. Gomes, G.B. Costa, G.W. Silva, and G. Véras, "Determination of biodiesel content in biodiesel/diesel blends using NIR and visible spectroscopy with variable selection", Talanta, vol. 87, pp. 30-34, 2011. [http://dx.doi.org/10.1016/j.talanta.2011.09.025] [PMID: 22099644]

[12] M. Fontalvo-Gómez, J.A. Colucci, N. Velez, and R.J. Romañach, "In-line near-infrared (NIR) and Raman spectroscopy coupled with principal component analysis (PCA) for in situ evaluation of the transesterification reaction", Appl. Spectrosc., vol. 67, no. 10, pp. 1142-1149, 2013.

[http://dx.doi.org/10.1366/12-06729] [PMID: 24067570]

[13] P. Felizardo, P. Baptista, M.S. Uva, J.C. Menezes, and M.J. Neiva Correia, "Monitoring biodiesel fuel quality by near infrared spectroscopy", J. Near Infrared Spectrosc., vol. 15, pp. 97-105, 2007. [http://dx.doi.org/10.1255/jnirs.714]

[14] S.M. de Lima, B.F.A. Silva, D.V. Pontes, C.F. Pereira, L. Stragevitch, and M.F. Pimentel, "In-line monitoring of the transesterification reactions for biodiesel production using NIR spectroscopy", Fuel, vol. 115, pp. 46-53, 2014. [http://dx.doi.org/10.1016/j.fuel.2013.06.057]

[15] R.R. de Oliveira, K.M.G. de Lima, R. Tauler, and A. de Juan, "Application of correlation constrained multivariate curve resolution alternating least-squares methods for determination of compounds of interest in biodiesel blends using NIR and UV-visible spectroscopic data", Talanta, 
vol. 125 , pp. $233-241,2014$.

[http://dx.doi.org/10.1016/j.talanta.2014.02.073] [PMID: 24840439]

[16] P. Felizardo, P. Baptista, J.C. Menezes, and M.J.N. Correia, "Multivariate near infrared spectroscopy models for predicting methanol and water content in biodiesel", Anal. Chim. Acta, vol. 595, no. 1-2, pp. 107-113, 2007. [http://dx.doi.org/10.1016/j.aca.2007.02.050] [PMID: 17605989]

[17] T. Izida, L. Bussler, J.R. Silva, L.H.C. Andrade, E. Simionatto, and E.L. Simionatto, "On-line in situ monitoring of the soybean oil and ethanol transesterification reaction by fluorescence spectroscopy", Fuel, vol. 145, pp. 109-115, 2015. [http://dx.doi.org/10.1016/j.fuel.2014.12.077]

[18] S. Pinzi, F. Alonso, J. García Olmo, and M.P. Dorado, "Near infrared reflectance spectroscopy and multivariate analysis to monitor reaction products during biodiesel production", Fuel, vol. 92, pp. 354-359, 2012. [http://dx.doi.org/10.1016/j.fuel.2011.07.006]

[19] S.A. Moreira, J. Sarraguça, D.F. Saraiva, R. Carvalho, and J.A. Lopes, "Optimization of NIR spectroscopy based PLSR models for critical properties of vegetable oils used in biodiesel production", Fuel, vol. 150, pp. 697-704, 2015. [http://dx.doi.org/10.1016/j.fuel.2015.02.082]

[20] A.B.F. Câmara, L.S. de Carvalho, C.L.M. de Morais, L.A.S. de Lima, H.O.M. de Araújo, and F.M. de Oliveira, "MCR-ALS and PLS coupled to NIR/MIR spectroscopies for quantification and identification of adulterant in biodiesel-diesel blends", Fuel, vol. 210, pp. 497-506, 2017. [http://dx.doi.org/10.1016/j.fuel.2017.08.072]

[21] R. Richard, B. Dubreuil, S. Thiebaud-Roux, and L. Prat, "On-line monitoring of the transesterification reaction carried out in microreactors using near infrared spectroscopy", Fuel, vol. 104, pp. 318-325, 2013. [http://dx.doi.org/10.1016/j.fuel.2012.07.054]

[22] C.L. Cunha, A.S. Luna, R.C.G. Oliveira, G.M. Xavier, M.L.L. Paredes, and A.R. Torres, "Predicting the properties of biodiesel and its blends using mid-FT-IR spectroscopy and first-order multivariate calibration", Fuel, vol. 204, pp. 185-194, 2017. [http://dx.doi.org/10.1016/j.fuel.2017.05.057]

[23] F.V.C. de Vasconcelos, P.F.B. de Souza Jr, M.F. Pimentel, M.J.C. Pontes, and C.F. Pereira, "Using near-infrared overtone regions to determine biodiesel content and adulteration of diesel/biodiesel blends with vegetable oils", Anal. Chim. Acta, vol. 716, pp. 101-107, 2012. [http://dx.doi.org/10.1016/j.aca.2011.12.027] [PMID: 22284883]

[24] Z.S. Baird, and V. Oja, "Predicting fuel properties using chemometrics: A review and an extension to temperature dependent physical properties by using infrared spectroscopy to predict density", Chemom. Intell. Lab. Syst., vol. 158, pp. 41-47, 2016. [http://dx.doi.org/10.1016/j.chemolab.2016.08.004]

[25] R.M. Balabin, R.Z. Safieva, and E.I. Lomakina, "Gasoline classification using near infrared (NIR) spectroscopy data: Comparison of multivariate techniques", Anal. Chim. Acta, vol. 671, no. 1-2, pp. 27-35, 2010. [http://dx.doi.org/10.1016/j.aca.2010.05.013] [PMID: 20541639]

[26] M.H.M. Killner, Y. Garro Linck, E. Danieli, J.J.R. Rohwedder, and B. Blümich, "Compact NMR spectroscopy for real-time monitoring of a biodiesel production", Fuel, vol. 139, pp. 240-247, 2015. [http://dx.doi.org/10.1016/j.fuel.2014.08.050]

[27] V. Gaydou, J. Kister, and N. Dupuy, "Evaluation of multiblock NIR/MIR PLS predictive models to detect adulteration of diesel/biodiesel blends by vegetal oil", Chemom. Intell. Lab. Syst., vol. 106, pp. 190-197, 2011. [http://dx.doi.org/10.1016/j.chemolab.2010.05.002]

[28] A. Palou, A. Miró, M. Blanco, R. Larraz, J.F. Gómez, T. Martínez, J.M. González, and M. Alcalà, "Calibration sets selection strategy for the construction of robust PLS models for prediction of biodiesel/diesel blends physico-chemical properties using NIR spectroscopy", Spectrochim. Acta A Mol. Biomol. Spectrosc., vol. 180, pp. 119-126, 2017. [http://dx.doi.org/10.1016/j.saa.2017.03.008] [PMID: 28284157]

[29] L.C. Gontijo, E. Guimarães, H. Mitsutake, F.B. De Santana, D.Q. Santos, and W. Borges Neto, "Quantification of soybean biodiesels in diesel blends according to ASTM E1655 using mid-infrared spectroscopy and multivariate calibration", Fuel, vol. 117, pp. 1111-1114, 2014. [http://dx.doi.org/10.1016/j.fuel.2013.10.043]

[30] M.J.C. Pontes, C.F. Pereira, M.F. Pimentel, F.V.C. Vasconcelos, and A.G.B. Silva, "Screening analysis to detect adulteration in diesel/biodiesel blends using near infrared spectrometry and multivariate classification", Talanta, vol. 85, no. 4, pp. $2159-2165,2011$. [http://dx.doi.org/10.1016/j.talanta.2011.07.064] [PMID: 21872073]

[31] S.P. Schale, T.M. Le, and K.M. Pierce, "Predicting feedstock and percent composition for blends of biodiesel with conventional diesel using chemometrics and gas chromatography-mass spectrometry", Talanta, vol. 94, pp. 320-327, 2012. [http://dx.doi.org/10.1016/j.talanta.2012.03.050] [PMID: 22608455]

[32] S. Soares, W.R. Melchert, and F.R.P. Rocha, "A flow-based procedure exploiting the lab-in-syringe approach for the determination of ester content in biodiesel and diesel/biodiesel blends", Talanta, vol. 174, pp. 556-561, 2017. [http://dx.doi.org/10.1016/j.talanta.2017.06.053] [PMID: 28738622]

[33] A. Paul, B. Bräuer, G. Nieuwenkamp, H. Ent, and W. Bremser, "A validated near-infrared spectroscopic method for methanol detection in biodiesel", Meas. Sci. Technol., vol. 27, p. 065002, 2016. [http://dx.doi.org/10.1088/0957-0233/27/6/065002] 
[34] E. Guimarães, L.C. Gontijo, H. Mitsutake, F.B. Santana, D.Q. Santos, and W. Borges Neto, "Quantification of Ethanol in Biodiesels Using Mid-Infrared Spectroscopy and Multivariate Calibration", Ind. Eng. Chem. Res., vol. 53, pp. 13575-13580, 2014. [http://dx.doi.org/10.1021/ie502067h]

[35] J.S. Oliveira, R. Montalvão, L. Daher, P.A.Z. Suarez, and J.C. Rubim, "Determination of methyl ester contents in biodiesel blends by FTIRATR and FTNIR spectroscopies", Talanta, vol. 69, no. 5, pp. 1278-1284, 2006. [http://dx.doi.org/10.1016/j.talanta.2006.01.002] [PMID: 18970715]

[36] R.M. Balabin, and S.V. Smirnov, "Variable selection in near-infrared spectroscopy: benchmarking of feature selection methods on biodiesel data", Anal. Chim. Acta, vol. 692, no. 1-2, pp. 63-72, 2011. [http://dx.doi.org/10.1016/j.aca.2011.03.006] [PMID: 21501713]

[37] W. Zhang, W. Yuan, X. Zhang, and M. Coronado, "Predicting the dynamic and kinematic viscosities of biodiesel-diesel blends using midand near-infrared spectroscopy", Appl. Energy, vol. 98, pp. 122-127, 2012. [http://dx.doi.org/10.1016/j.apenergy.2012.03.013]

[38] R.F. Sales, R. Vitale, S.M. de Lima, M.F. Pimentel, L. Stragevitch, and A. Ferrer, "Multivariate statistical process control charts for batch monitoring of transesterification reactions for biodiesel production based on near-infrared spectroscopy", Comput. Chem. Eng., vol. 94, pp. 343-353, 2016. [http://dx.doi.org/10.1016/j.compchemeng.2016.08.013]

[39] M. Bonato, A. Valério, J.V. Oliveira, D. de Oliveira, and A. Bolzan, "Midinfrared spectroscopy and partial least-squares model as an analytical method for biodiesel and glycerol monitoring", Ind. Eng. Chem. Res., vol. 57, pp. 990-996, 2018. [http://dx.doi.org/10.1021/acs.iecr.7b04074]

[40] E.L.M. Arias, P.F. Martins, A.L.J. Munhoz, L. Gutierrez-Rivera, and R. Maciel Filho, "Continuous synthesis and in situ monitoring of biodiesel production in different micro fluidic devices", Ind. Eng. Chem. Res., vol. 51, pp. 10755-10767, 2012. [http://dx.doi.org/10.1021/ie300486v]

[41] L. Canoira, M. Rodríguez-Gamero, E. Querol, R. Alcántara, M. Lapuerta, and F. Oliva, "Biodiesel from low-grade animal fat : Production process assessment and biodiesel properties characterization", Ind. Eng. Chem. Res., vol. 47, pp. 7997-8004, 2008. [http://dx.doi.org/10.1021/ie8002045]

[42] M.A. Dubé, S. Zheng, D.D. McLean, and M. Kates, "A comparison of attenuated total reflectance-FTIR spectroscopy and GPC for monitoring biodiesel production", J. Am. Oil Chem. Soc., vol. 81, pp. 599-603, 2004. [http://dx.doi.org/10.1007/s11746-006-0948-x]

[43] M.G. Trevisan, C.M. Garcia, U. Schuchardt, and R.J. Poppi, "Evolving factor analysis-based method for correcting monitoring delay in different batch runs for use with PLS: On-line monitoring of a transesterification reaction by ATR-FTIR", Talanta, vol. 74, no. 4, pp. 971-976, 2008. [http://dx.doi.org/10.1016/j.talanta.2007.08.004] [PMID: 18371736]

[44] G.F. Zagonel, P. Peralta-Zamora, and L.P. Ramos, "Multivariate monitoring of soybean oil ethanolysis by FTIR", Talanta, vol. 63, no. 4, pp. 1021-1025, 2004.

[http://dx.doi.org/10.1016/j.talanta.2004.01.008] [PMID: 18969529]

[45] E.M. Paiva, J.J.R. Rohwedder, C. Pasquini, M.F. Pimentel, and C.F. Pereira, "Quantification of biodiesel and adulteration with vegetable oils in diesel/biodiesel blends using portable near-infrared spectrometer", Fuel, vol. 160, pp. 57-63, 2015. [http://dx.doi.org/10.1016/j.fuel.2015.07.067]

[46] R.M. Correia, E. Domingos, V.M. Cáo, B.R.F. Araujo, S. Sena, L.U. Pinheiro, A.M. Fontes, L.F.M. Aquino, E.C. Ferreira, P.R. Filgueiras, and W. Romão, "Portable near infrared spectroscopy applied to fuel quality control", Talanta, vol. 176, pp. 26-33, 2018. [http://dx.doi.org/10.1016/j.talanta.2017.07.094] [PMID: 28917750]

[47] P. Verma, and M.P. Sharma, "Review of process parameters for biodiesel production from different feedstocks", Renew. Sustain. Energy Rev., vol. 62, pp. 1063-1071, 2016.

[http://dx.doi.org/10.1016/j.rser.2016.04.054]

[48] Y. Warabi, D. Kusdiana, and S. Saka, "Reactivity of triglycerides and fatty acids of rapeseed oil in supercritical alcohols", Bioresour. Technol., vol. 91, no. 3, pp. 283-287, 2004. [http://dx.doi.org/10.1016/S0960-8524(03)00202-5] [PMID: 14607488]

[49] B.V. Sitoe, A.D.V. Máquina, F.B. de Santana, L.C. Gontijo, D.Q. Santos, and W. Borges Neto, "Monitoring of biodiesel content and adulterant presence in methyl and ethyl biodiesels of jatropha in blends with mineral diesel using MIR spectrometry and multivariate control charts", Fuel, vol. 191, pp. 290-299, 2017. [http://dx.doi.org/10.1016/j.fuel.2016.11.078]

[50] G. Mendow, N.S. Veizaga, B.S. Sánchez, and C.A. Querini, "Biodiesel production by two-stage transesterification with ethanol", Bioresour. Technol., vol. 102, no. 22, pp. 10407-10413, 2011. [http://dx.doi.org/10.1016/j.biortech.2011.08.052] [PMID: 21920733]

[51] B. Freedman, E.H. Pryde, and T.L. Mounts, "Variables affecting the yields of fatty esters from transesterified vegetable oils", J. Am. Oil Chem. Soc., vol. 61, pp. 1638-1643, 1984. [http://dx.doi.org/10.1007/BF02541649] 
[52] P. Bondioli, and L. Della Bella, "An alternative spectrophotometric method for the determination of free glycerol in biodiesel", Eur. J. Lipid Sci. Technol., vol. 107, pp. 153-157, 2005

[http://dx.doi.org/10.1002/ejlt.200401054]

\section{(C) 2018 Gelinski et al.}

This is an open access article distributed under the terms of the Creative Commons Attribution 4.0 International Public License (CC-BY 4.0), a copy of which is available at: https://creativecommons.org/licenses/by/4.0/legalcode. This license permits unrestricted use, distribution, and reproduction in any medium, provided the original author and source are credited. 